\title{
A INSERÇÃO DO TEMA LIDERANÇA NAS DISCIPLINAS DE ADMINISTRAÇÃO EM ENFERMAGEM DE UMA FACULDADE PARTICULAR DA CIDADE DE SÃO PAULO 1998 A 2004
}

The Leadership Theme Insertion in the Nursing Administration of a Private College in São Paulo City 1998 to 2004

La Inserción del Tema Liderazgo en las Disciplinas de Administración en

Enfermería de una Facultad Particular de la Ciudad de São Paulo 1998 a 2004

Luinar ReginaConstantino

MariaCristinaSarna

\section{Resumo}

Para descrever aevdução da abordagem da Liderança nos programas des disaiplines deAdhinistração em Erfermagem

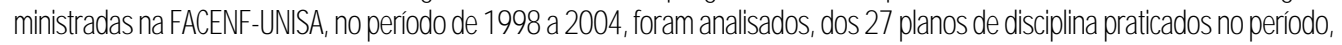
os djetivoseas estrdé́jis deensino-aprendzagemedeaveliação. Otrdamentoutilizadb, análisededsarsodeBardn

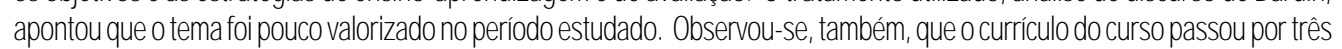

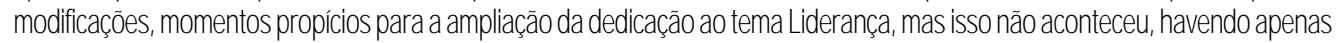

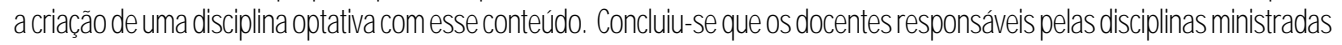

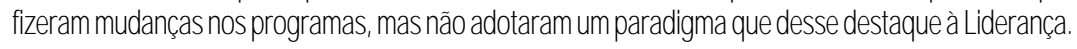

Pałaras dhave Adhinistração. Etfermagem Ensino. Hstónia Liderança.

Abstract

With the dbjective of describetheevolution of leadership Con el objetivo de describir la evoludón del abordajeda approach of theplans in theeightdisaplines, dedicated to Liderazgoenlos programasdelas odhodsajlines dedicadas nursingadhinistrdion, nagistrstedatnursingthecollege of alaAdhiristradóndelaEfermeria, ministradesenlaFaaltad Utisaintheperiodfrom 1996 to2004, vereandyzedfrom deErfermeria dela Universidad UNSA en el periodo de 27plans ofdssiplines pradiaingintheperiod thedjectives, 1996 a 2004, fueron analizados, de los 27 planes de thestrategic of teading- leaming of to and of value, the disciplinas pradicados enese periodo, los dbjetivos, las uilized treatrent analysis of speech Bardin, point that estrdegias delaenseñarzazorendzajeydeeveluacón 1 leadashipthemenesnotmorhevelutedintheperiodstuded trataientoutilizadb-análisiscedsarsodeBardin apurtó it was obsened too that the a rial um of thecaursehas quee tema Liderazgo fué poco valorizado en el periodo passed by trree moments of the dhanges in the period estudiado. Seobservótambiénquee a mialo del arso studied propitious to improvethededication toleadership pesópor tres modificaciones, nomentos propicios parala theme, but itdd nothappen, remaining only thecreation of expansióndela dedicadónal tema Liderazgo, nas estono oneqpional dsciplinewiththis cortert, condusionves that oamió, consiguiéndoseapeneslacreacóndeunadsaplina academic staff responsilde for their dsapline nursing optativaconeseconterido. Conduyesequelos dbcentes adhiristrationnadedhangeintheplans, butnothauingin responsables por las disciplinas de Adrivitración en mindtheeductional drection paradgmwich couldstand Effermeriahicieronmudarzasenlosprogramas, mas noen attheleadershipthere

\section{Keyouds \\ Nursing Adhiristration Eduction Hstory. Leadership.}

direcióna un paradigraedicacional quediesedestaqued termiliderazgo.

Palabras dave: Effermería.Adhinistración Enser̃arza Hstoria Liderazgo. 
INTRODUÇÃO

O Curso de Graduação emEnfermagemda Faauldade de Enfermagem da Universicade de Santo Anaro (FACENF-UNSA), localizada na região sudbeste do muiápio de São Paulo, foi criado em1995, por meio da resolução CONSU 009/95 de 17/05/95, iniciou sua primera turma em 1996 e foi reconhedido pelo MEC medante a Portaria MEC 1880/ 2001.

O Projeto Pedagógico do Curso de Enfermagem, em vigor em 2005, assim como os que lhe antecederam, pressupõe atividades de ensino, pesquisa e extensão, tendo como dbjetivo do curso, "formar profissional enfermiro apto ao exerćcio da Enfermagemnas áreas assistencial, administrativa, de ensino e pesquisa, qualificandb-o para sua inserção no tercadb de trabalho(1)".

AFACEN-UNSA foi criada numa época de grandes transformações no ensino superior e, particulamente, no ensino de Enfermagem numa conjurtura emque fai patada a inserção de novos valores, fruto de umproces so de transformacões educacionais, comb os expressos ra Portaria MECNb. 1721, promilgada em15 de dezem bro de 1994, que fixau o mínimo de conteúdb e duração dos arsos de graduação em Erfertagem ${ }^{(1)}$.

Em 2001, entrou emvigor uma nova proposta arrialar, aprovada pelo Conselho Universitánio, como resposta às mudanças decorrentes da nova Lei de Dretrizes e Bases de Educação Nacional (1) , e, em2003, mais mudanças na distribuição da carga horária foramfeitas para contemplar o previsto nas Diretrizes Ciriaulares para o Curso de Graduação emEnferma$\mathrm{gem}^{(1)}$. Emambas, o arso tinha a duração de quatro anos e regime anual, dferente do primeiro projeto, aja duração era de anco anos.

Esses registros são inportates, nẽo apenes porquendrcarmo desendinito to da FACEN-UNSA nas porqueatorraramummodlo interessante pra estudb, una vez que fá criada emummonerto de transformações ra Educação, o que implicau emvérios descaios para o seu conpo dbcertee dscerte, qu, emmenos de dez anos de existênia viencarm

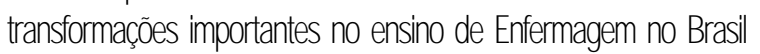
eprotagonizaramgrandes molanças a rialares.

$\mathrm{Nb}$ que diz respeito ao contaúdo de Adhinistração emEnfermagem deve-se registrar que, no primeiro a míalo, estefoi miristrado pela primera vez em1998, no terceiro ano, numa disciplina denominada " $\mathrm{Adm}$ nistração Aplicada à Enfermagem". O projeto peda gógico de então funcionou đé 2003, quando, no quinto ano, se lecionava una disciplina que abordava exdusivamente a ideologia da Qualidade, denominada "Controle de Qualidade", e se realizavamas ativida des de ensino prático sob supenisão de todo o conteúdo de Adrinistração emEnfermagem

Em2001, entrou emvigor umnovo a míalo, nas, para os ingressantes antes deste ano, continuourse a oferecer o a míalo antigo. Assim conviveu-se por dois anos comdois aumáculos diferentes. No novo modelo, ao qual aderiramos ingressantes em2000 e foraminseridos os ingressantes de 2001, passaram se a oferecer disaiplinas de Administração emErfermagememtodas as séries, numeradas de I a IV, conforme a etapa do aluno no aurso, tendo atividades práticas no terceiro e quarto ano, neste útimo como parte do estágio a rrialar. A disciplina de "Controle da Qualidade" do a míalo antigo foi extinta, e seu conteúdo, inconporado às demais. Emfunção desses acontedimentos, o ensino da disaiplina de Administra ção emEnfermagem passou a ocupar una posição de destaque, coma ampliação da carga horária de 680 ha para 816h/a

A compreensão destas transformaçães pode contribuir para a reffexão sdare o processo ed racional, emespecia o ensino da Adhiristração emEtermageme o desenndvinetto da habilidade emLidarança, term do preserte estud, que sempre fai umdescio para a Evermagem.

Stbeseque o ensin deAdhinistração ea páticagarenid de Erfermagentêmsues rázes, conod descreesestra ${ }^{(2)}$, nos

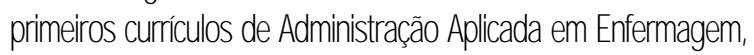
quand estes foramestudades ra pinera escda de Everma

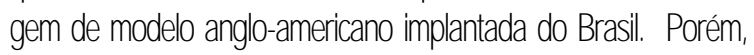

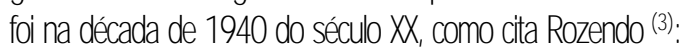

Qeaurertou o interesse pelas dividades desemperha cas pela enfermeriadh efe e pela formação deste profissiond, aja preoupação certrd ea o dendretto da ne cessidade da preparação pra o exerácio gerendal.

Recorheceurse que a necessidade do desenvdvinerto de habilidades de liderança e o preparo do aluno eramne cessários, desde a inserção das primeriras diplonadas no nercadb detrabalho, cono descrevemMatins eSarna ${ }^{(4)}$ : mitas vezes, enfermeiras recériformadas eramconvidadas a œa paremcargos de grandeimportânia para a Evferta geme para o sisterm de sáde

Coma evolução da dêndia da Administração, mitas teorias da liderança vierame se foram mas a Liderança, como categoria conceitual da prática gerencial, continuou a ser alvo de atenção, embora nemsempre bem definida nas primeiras pesquisas que deste tera se oaparam

Estudosos cono Siñ̃es e Fávero (5) afirmaramque a liderança é u mfenômeno gupal, que envolve um sistera de influência sodial de umindivíduo sobre 
os denais, é umprocesso coletivo, compartilhadb entre os derertos de umgrupo e, sendb assim fortde ce o gupo de trabalho, velorizandb as competênás indVidus, fazendb comque cada mentro recorheça o propósito e o significadb de seu trabalho.

Analisando a definição dessas autoras, questiona se: se a liderança é tão importante para o enfermèro, como ensinar a ser umlíder eficaz?

De acordo com Simões ${ }^{(6)}$,

umdbs desafios theis écono aprender a ser umlíder semnodlos efetivos de liderança emenfermagemnos quis os alunos possamse espel her ea fatta de qportur ricade que favoreça o desenndvimerto de habilidades de liderança, induindb a fatta de estímolos.

Em1977, Secaf (7) disatiu o porquê da falta de modles efetivos de liderança e a desvalonização da liderança emenfermagem A autora ponderou que todos os alunos aprendiama desenvolver habilidades no aidado de enfermagem, porém, habilidades referentes à liderança eramabordadas de formæ simplista, formando profissionais que não reconhediamsua im portância e não conseguiamassumir o papel de um enfermeiro líder. Após quese 28 anos desta publica ção, presumese que a situação pouco midu quando se lê o estudo de Siñ̃es e Fávero (8), datado de 2000, sobre a aprendizagemda liderança, no qual os discursos de enfermeiros recémformados que trabalhamemuma instituição hospitalar reforçama ne cessidade de se inserir o desenvolvimento de habilidades de liderança nos auríalos de graduação em enfermagem e ainda apontamque una pessoa mesmo despica de habilidades especiais, pode se tomar umlíder; faz-se então necessário que os órgãos formadores se sensibilizeme efetivemumprocesso ensino-aprendzagem o qual possibilite o desenvivinento de habilidades próprias da função.

Siñ̃es e Fávero ${ }^{(8)}$ reconhecem que as instituições de ensino têma responsabilidade e umpapel desafiador emformar e lançar, no mercado de trabar Iho, profissionais devidamente preparados para assut mir a função de liderar a equipe de enfermagem Já Galvão et al. ${ }^{(9)}$ emuma reflexão sobre a liderança do enfermeiro no séalo XX pergurtam a formação proporcionada pelas instituições de ensino é satisfatónia para o enfermeiro assumir a liderança requerida para o próximo século? De fato, Chaves ${ }^{(10)}$ dexa indícios de que há uma grande insatisfação quanto à formação proporcionada a esses futuros profissionais, ao recomendar a estudantes de enfermagem que serão fur turos líderes, que busquemincessanterente publica çes dientificas sdbre o terma Por atro ladb, sabermes que, cono aimamHga e Trevizan (11), "as pesquisas sobre lide rança ainda são escassas, næs emcrescerte desendvinen to, indcandb que precisanos de líderes que estejammetivar dos, comespárito cridivo para experinertar, compreender e praticar as habilidades de liderança".

Sabe-se porém que, a despeito do progresso observado na pesquisa em Enfermagem com a explosão no números de cursos de graduação e a ampliação da atuação gerendal do enfermeiro, ainda há poucos profissionais engajados na luta pelo ensino da liderança, embora esta problemática date de quase 40 anos, como pode ser constatado nos registros conticbs nos arais do XXCongesso Braileiro de Enfermagem, relizadb emPeife, en1968, ajo ten certrd fó “Edrar para a Lidarança" (12), no qua foramfätes digunas recontendax̧ões cona Qe, ros a míalos das Escolas de Enermagen, ckeser dada êffase aos aspectos de liderança profissional, que promerna formaz̧ão cb alum para as responsabilicades que Ihe caberão ro equecionanerto dos prodlenæs de liderança de sua prơfissão e que promovamaursos de etensão para effermiriss sobre os aspectos de liderança.

Cumpre ainda rendionar a criação de umprograna menda, lideradb pelo Conselho Internediond de Evfermeriras (ICN) (13) e patrocinadb pela Fundação Kellog denominadb "Liderazgo para ed contbio", a liderança para modança com ińdio em1996, que abrangeu os páses da Anérica Latira e Caibecomo dgietivo de contribuir pera nethorar os senvicos de saúde, atranés de programas de formaşão emliderança para os enfermeros e enfermeros gestores; e propor mudanças na preparação e formação do enfermeiro nos diversos páses, dante dos descios que se originavamdas rápidess e irtensas mudançes no setor de đenção à saúde.

En2005 ocorreu o 4 Encontro Nacional dos Docentes de Administração emEnfermagem ${ }^{(14)}$, realizado durante o 560 Congresso Brasileiro de Enfermagem no qual foi dediddo que, dentre as competênias e habilidades do egresso de arso de graduação em enfermagem está: Desenvolver e aplicar os prinápios de liderança nos processos de trabalho da equipe de saúde e de enfermagem

Observa-se que tambémos manuais de normas e procedimentos do Senviço de Enfermagem das instituições de saúde, ajo valor foi reefimado pelos esforços empreendidbs para a conquista de Acreditação dos Senviços Saúde, atribuemo papel de líder ao enfermeiro, e os organogramas refletemuma posição estratégica desse profissional na hierarquia institudional. A percepção dessa posição que o effermeiro oapa e as transformaçães observadas no trabalho emsaúde 
acabampor impulsionar o aluno a buscar noves nat neiras de aprendizagem proaurando posicionar-se de acordo como perfil do mercado de trabalho atual e preparar-se para o desemperho profissional.

Do ponto de vista das autoras do presente estudo, repensar a formação desses profissionais pode ajudar a construir uma nova proposta para o processo de ensino-aprendzagem e é por isso que os atuais e futuros profissionais predisam perceber e conquistar o avanço, no sentido de transformar o processo edtr cacional e intenvir junto aos docentes para que essa mudança seja completa Nmt $^{(15)}$ temrazão quando afirma que a Educação é uma prática social que se desenvolve de maneira semelhante a umprocesso de trabalho, compondo-se de mediações entre docente e discente, para que o processo seja intencionalizado. Essas transformações originamse, também de valores e interesses econômicos, políticos e sociais dos quais a Enfermagemdeve fazer parte e ajas transformacões deve acomparhar.

Comisso, condui-se que énecessário disatir e refletir sobre o ensino da Liderança emE fremagem Nesse sent-

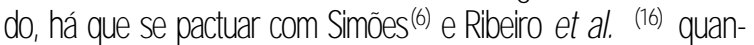
do ressatta que estu dar liderança é tão necessário e desafia dor quanto ariscad, pais sabernes que rẽo existemfớmet las ou receitas predeterminadas.

Foi assimque, perante as vivêndias na FACENFUNSA como discente e dbcente, junto aos outros alur nos e profissionais das mais diversas instituições de saúde emque se realizava a prática de ensino de Administração, foi possível perceber, nas alturas das organizações envolvidas, hábitos, crenças e diferentes valores morais que envolviama Liderança, o que incomodou de forma a despertar a necessidades de proaurar bases para tentar entender essas relações profissionais Foi etrão que se percebeu a possibilidade da existênaja de una lacu na no processo ed cadiond nedado por dbcertes e dscertes, represertada justanerte pela par ca exdiataçãa, emespedial nes dsajplines de Adhinistração emErfermagem, sobre as diversas formas de desendiver habilidades de liderança

Hbje, como futura profissional de Enfermageme docente antiga, preocuparro-nos comas oportunicades de formação atualmente oferedidas e as que o serão aos futuros alunos, já que se constata que a preparação profissional ainda é influenciada por paradignas que são descritos por Sena \& $a$. (17), ao analisaremo projeto pedagógico de dez escolas de ensino superior de Enfermagem como ainda sustentados no modelo "flexneriano" ou, no seu corolário indssolúne, o proposto no relatónio Coodhrak, conduídb em 1910, nos Estadbs Utidbs cla Arética db Nate
Vale salientar que a valonização insuficiente do en sino da Liderança temsuas conseqüênias registradas por Nitz e Clampone ${ }^{(18)}$, que constataramque o professor effermero, mais que ensinar a profissão, é quem delimita no aluno atitudes e conceitos que tendema se refletir na prática profissional e na enfermagem como profissão.

Faria e Casagrande ${ }^{(19)}$ afimaramque os maiores desafios do século atual estão emaprender a viver juntos neste mundb globalizado, ea Educação elerge como o grande trunfo, por possibilitar o desenvolvimento contínuo de pessoas e de sociedades. Hije se fala mito emuma Educação que propicie a formação de profissionais áticos e flexiveis. Cono relatam Comes e Casagrande ${ }^{(20)}$ o cenário educacional não será uma educação para o conformismo, mas será uma educação voltada para a liberdade e a autonomia denominada como 'autura reflexiva'.

Assim vêse que há esforços de educadores para lançar, no mercadb de trabalho, profissionais engajados e compromissados comessa mudança Nota-se tam bémque vários estudiosos manifestarampreocupa ções acerca do preparo do enfermeiro para liderança nos cursos de graduação e cresce o interesse em obsenvar como este ensino se dá

Una das altemativas para mergulhar no exame dessa problenática poderia ser a análise dos programas das disciplinas de Administração emEnfermagem tal como fai realizado por Sama ${ }^{(2)}$ e Nitz ${ }^{(15)}$, ao descreveremo processo da evolução da disaiplina de Adrinistração emEnfermagememseus estudos.

Assim a opção pelo estudo dos planos da disaiplina de Administração em Enfermagem deve-se pela oportunidade de resgatar a essêndia do fenômeno vivenciado pelos atores do processo ensino-aprendzagem da época estudada, pois, considerando Bordenave e Pereira (21):

todo plano é estabelecido por objetivo definido, estratégia de ensino e avaliação, no qual objetivos são corhecimentos, habilidades intelectuais, atitur des e valores, alémde habilidades motoras, que devernser desenvolvidos nos alunos.

Já estrdégade ensinoé pra os a tores atadbs's2) conto facilitar a passagemdos alunos da situação emque se encontramaté alcançaremos objetivos fixados [...] e avaliação é evidenciar se o objetivo foi alcançado e emque grau foi alcançado.

É por isso que a formação do aluno é constrúcla e orientada por uma míalo ao qual, segundo Sena et al. (17), devemser incorporadas competêndias para que, pela definição, as demandas do mercado sejam 
consideradas. MBS, na opinião de Abreu e Mbsetto (22), não se pode negar que o arso proara formar profissionais segundo a linha, o espínito, os prinápios e os propósitos da instituição da qual faz parte.

Assim ressalta-se a importância de resgatar o tema Liderança, nos planos das disciplinas de Administração em Enfermagem da FACENF-UNSA dada a sua converiêndia não só para a compreensão da histónia da instituição, mas, prinapalmente, para a apreensão da realidade de transformações ocomidas na História do Ensino da Liderança emEnfermagem nos útitiros anos.

\section{OBJ ETIVO}

Descrever a eldução da inßerção do term Lidkrança nos progranas des dsaiplines de Adhinistração em Erfermagem do Curso de Graduação em Erfermagemda Faa ldade de Evfermagem da Utiversidade de Sarto Anaro, no período de1998a2004.

\section{METODOLOGIA}

Foi realizadb umestudb qualitativo que, segundo Mnayo (23), são aqueles capazes de incorporar a questão do significadb e da intencionalidade como inerentes aos atos, às relaçães e às estuturas sociais, apoiado no paradignæ fenomenológico.

Para atingir o dojetivo, foramsolicitadbs e obtidos todos os vinte e sete planos das disciplinas de Adhiristração emEnfermagemreferentes aos anos de 1998 a 2004, junto à Secretaria da FACENF-UNSA Os dbar nentos foramcopiados, e foi solicitada a autenticação das cópias à Diretoria da FACENF-UNSA que, ao fazêlo como conhedimento de sua finalidade, atorizou a rea liação da pesquisa. Oprojeto não fai sulumetidb ao Gmitê de Éica emPesquisa comseres Humanos por tratar-se de pesquisa embanco de dadbs.

Emseguida, procedeurse à escolha dos itens que cabiampara análise, obsenvando-se aqueles no qual se apresentava a intencionalidade do professor, percebidas no uso de verbos, como empregado na enunaiação dos itens objetivos, estratégias de ensino e de avaliação, nos planos de disajplina.

Para compreensão do fenômeno emestudb, esta pesquisa tomou como ponto de partica o trabalho de Sarna ${ }^{(2)}$, optando-se, diferenterente do empregado na obra citada, pela análise de conteúdo, segundo Bardin $^{(24)}$, mais adequada para a análise dos textos contidos nos doaumentos analisados. A retodologia adotada é definida como um conjunto de técricas de análise de comnicaçãa, visando obter, por procedi- mentos sistenáticos e objeivos de descrição do conteúdo das mensagens, a compreensão de sua intencionalidade Segundo esta autora, este método tempor dgjetivo chegar à inferência, a partir dos efeitos de superfície de u ma estrutura profinda, [pela qual] esta técrica tenta estabelecer ligações entre a siturção na qual o sujeito se encontra e as manifestações semântico-sintáticas da superfíde disarsiva.

Mnayo ${ }^{(23)}$ alerta que o objeivo da análise de conteúdb é realizar uma reflexão geral sobre as condções de produção e apreensão da significação de textos produzidos nos mais diferentes campos. Assim tomourse como textos, os planos de disaiplina, considerando-se que os mesmos são a expressão do pensamento do professor sobre o fenômeno.

Os procedimentos para a exeaçãa da técrica de análise de discurso, percorridos na realização do presente trabalho, compreenderamas seguintes fases (24): preparação do discurso, quando se busca a dependência das frases através do desmentbramento por operações lingǘsticas, como substituição de anáforas, recuiperação das preposições latentes e restabelecimento da ordem corrente da frase, procurando encontrar dependências funcionais; dassificação das relações binárias, sendo codificadas no momento emque se realiza a análise automática, surgindo, assim blocos de pré-categorias; reagnuparento dos pares de précctegorias, quando se analisamesses pares embusca das categorias de pensamento, através das condições de proximidade semântica e se estiverem ligados por dependêndias funcionais idênticas a dois outros enunciados, eles próprios próximos umdo autro; e construção de una tábua de convergência entre as categorias de pensamento, expondo com que freqüência essas categorias aparecemno texto, de aja descrição se depreende a intencionalidade do disarso.

Depois disso, os achados foramconfrontados com - que registra a literatura dentifica existente sobre o terma, a fimde permitir a sua disaussão e emergir a compreensão do fenômeno.

\section{RESULTADOS E DISCUSSÃO}

Aanálise dos 27 programas das disciplinas pratica dos no períodb estudado revelaram, no item Objeivos, trezentos e quarenta e dbis disarsos, cento e dezenove domínios ou pré categorias de pensamento e trinta e nove hiperdomínios ou categorias de pensamento. Os itens colhidos sob o tútulo objeivos estão apresentados na Figura 1 emanexo, demonstrando comque freqüênaia aparece o termo Liderança nos planos da 
disaiplina de Administração emEnfermagem indicando os comportamentos finais que se espera que se jamexibidos pelos alunos. A Aesse propósito, lembra se que Abreu e Maselto (22) referemse a tais comportamento como expressão de corheamentos ou de atjtudes e habilidades que, antes de arsar a disciplina, o aluno não era capaz de realizar.

A freqüêndia comque apareceramos diferentes rútodos de Avaliação da Aprendizagemaplicados pelos docentes responsáveis pelas disaiplinas revela como se comportava o professor sobre a aprediação da ca pacidade que o aluno adquiriu para desemperhar o papel de umlíder, mo decorrer do processo educacional. É propício comentar que algumas estratégias de avaliação foramredigidas semo emprego explíito do verbo, difialtando a perceppção da intencionalidade do professor, o que obrigou apenas à sua ditação como tipo de avaliação a ser aplicado ao aluno, mas isso não inviabilizou a análise.

Ao contemplar os resultados obtidos, ficou evidente que os planos das disaiplinas de Administração em Enfermagemestudados valorizarampreferendialmente as Teorias de Administração e a Qualidade da Assistência de Enfermagem A preocupação como primeiro tópico revela que os docentes dâo ênfase às Teorias de Administração emgeral semconexão aparente coma sua aplicabilidade à Administração emEnfermagem Verificourse que essa abordagemé freqüente emquase todos os anos, indicando umauríallo estático. Tambámse verificou que o segundo tópico mais freqüente nos planos de disciplina apontou pre dominantemente para ummodelo de ensino voltado para a área de atuação intra-hospitalar, modlo este que, segundo Clampone et al. ${ }^{(25)}$ não mais responde às demandas presentes no nosso contexto da saúde".

Ofoco do presente estudb, o terma Liderança, apa receu comuma pequena ocorrência de freqüênda, indicando a desvalorização deste terma pelos docentes. É oportuno comentar que, combase nas Diretrizes Curialares para o Uurso de Graduação emEnfermagemdo MECe o que contempla o projeto pedagógico do arso da FACENF-UNSA o perfil do profissional a ser desenvolvido por seus graduandos indu' Liderança de equipe de saúde (1). Assim podese afirmar que há umdescompasso entre o que se propõe e o que se efetiva, já que a Liderança parece não ser o que se pretendia ensinar aos alunos.

Ressaltese ainda que os graduados nesta faculdade podemestar saindo despreparados para desem perhar a função de liderar uma equipe de enfermagem A esse propósito, podese indicar o que revela umestudo realizado por Casa ${ }^{(26)}$ comalunos do $4^{\circ}$ ano emestágio a riaular da FACEN-UNSA sobre o uso de portfólios como instrumento de reflexão na formação de umlíder, emque ficou evidente o desprepero desses na habilidade emfoco. A autora categonizou as fases da vivência dos alunos na prática, estabele cendo a primeira fase como insegurança, que definiu como incertezas, confusão e medo emrelação às atividades a desemperhar e falta de preparo para o de semperho da função. Chama à atenção que a presença de confusão e redo na fase inicial, que são reações aceitas para qualquer profissional ao se deparar como desconhedido, se somamincertezas e falta de preparo, que não são aceitas do profissional ou do futuro enfermeiro no fimdo processo de formação, que se depara com umanbiente no qual terá que se posicionar como umlíder.

É desejável que alunos universitários do útimo ano terhamadquirido habilidades durante o processo de formação e estejamcapaditados a desenvolver e pra ticar as restras no final do arso, no período dedica do à prévivênda do papel profissional. Para fortale cer esse argumento, deve-se lembrar que Abreu e Maselto(22) definemestágio como una estratégia que coloca o aluno emconfronto comsituações reais, em que os alunos têmhabilidades suficientes para perce ber situações e tomar dedisões.

Voltandb ao estudb de Casa (26), na quarta fase, que esta define comro aquisição de competêndias de lide rança, a autora afimmu que esses graduandbs se tornaramlíderes reflexivos. Contrastando coma ocorrêndia de freqüêndia comque aparece o termo Lide rança, nos planos das disáplinas de Adninistração em Evfermagem maior nos programas do $3^{\circ}$ ano e vaga mente no 4 ano, fica a indagação: como de acabou por se tomar esse líder reflexivo? Lembrese que, no úlimo ano, os alunos têmaproximadamente 42 dias de estágio, o que equindea 720 horas, canforme os planos cla disajplina dermonstram, ocorrendb aparentemente una superação tota das dfia lokdes par pate destes gadurdos, quando a autora deixa implíito que emapenas pouco mais de ummês, os graduandos conseguiramtomar-se líderes. Seria isto possivel? Quis rearsos de aprendza gemteriamsidb utilizadbs?

Sabese que a farmação db efermiro se completa ecan tina ra prática labord, eque exs exeriênias da gradação rão são u matestadb incontestável de que as competênias para liderar foramplenanerte desendvidas, nas, nesmo assim fica a inquietação darte do contrastesúbito ertre un $A$ posiçã e a tra apontades por Casa ${ }^{26}$ ao longo de tão reldiva nerte arto períocb de prática dscerte sob superisãa 
Nas estratégias de ensino-aprendizagem podese observar que predominaram aquelas em que 0 professor dirige as atividades dos alunos, com preleção dialogada, estudo dirigido e painés, dentre outras. Considerando que as estratégias induídas nesses planos não favorecem o desempenho de habilidades de umlíder, como o aluno estaria se preparando para liderar?

Qs est dosos da Ldática afimamque compete as dbcente despertar interesse, dranisto eatonomia mo dum, o que representaria umpreparo inicial para o desemperho da rablidadede lickar. Ao aportar, nos planos de Adhinistração emErfermagem, aulas expositives, seminánios e estudos drigidos como estrdégias preferendas, condu-seque estes rẽo con Nêmpara que o aluno desemperhe habilidades de liderança e se desen ohvemnessa prática

Sobre isso érdevate sliettar que des dess três atoras de u mtrabalho pudicadb soldre estratégias inovedoras no ensino de Adninistração aplicada à Effermagem cainidarten de Adhinistração emEtermagem da FACAN-UNSA Essas

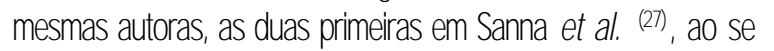
posicioneremsobre os nétodos de ensino tradicionais, dedararamque

O emprego desses reaursos pode até ser eficaz quando destinado ao ensino de aspectos biológicos ou de intenvenções padronizadas, mas tal racioánio não se aplica quando se trata de aspecto menos objeivo, como aqueles que compõemo conteúdo da disciplina de Adninistração emEnfermagem

As autoras ainda ressaltaram em1999, que inover na escolha de estratégias-ensino aprendizagem pressupõe o reposicionamento do professor de Advinistração emEnfermagemfrente às mulanças que ocorremna profissão, assumindo a responsabilidade de preparar enfermeiros capazes de enfrentar os desafios do próximo sédula Ao olhar para estratégias de ensino-aprendizagem indicadas nos planos, pode se afimar que essas autoras reconhecem a importância, mas se abstêmdo seu reposicionamento diante das mudanças, não assumindo a responsabilidade de formaremenfermerros capazes, conforme descrevemos seus planos das disaiplinas de Administração emEnfermagem

Há grandes esforços dos docentes que lecionam este conteúdo emoutros aursos para encontrar saídas para esse impasse Refletindo sobre essas $\mathrm{mr}$ danças, Takahashi e Peres ${ }^{(28)}$ pronunciamse desta forma: os educadores sempre assumiramuma estra tégia moldada de acordb comos padrões [...] Assim quebrar velhos paradigmas e assumir novos paradignas é um desafio para o docente, que contribuirá para o ensino da Enfernagem

Ao analisar os planos de disciplina do aurso em foco, pode-se afimar que este desafio ainda não foi superado, mito embora apenas o seguimento dos egressos formados no novo a míalo possa revelar isso. Como só há seguimento de egressos do primeiro a níalo praticado na FACEN-UNSA não é possível afimar nemque foramou que não foramformados líderes no novo modelo a rialar praticado.

Considerourse tambémque os docentes confundemestratégia de avaliação de aprendizagemcom estratégias de ensino, quando, por exemplo, entre outras ações propõema daboração de ensaios com exerácios de síntese e aplicação dos conhedimentos aprendidos, mas os empregampara aferir o alcance dos dijeivos da dsajplina elhes dribuemnatas que com põemo conjunto de reios de avaliação dotadas com essa finalidade Bordenave e Pereira ${ }^{(2)}$ ensinamque há quatro formas de aveliação: prova ord, prova prática, a dissertação qu ensaio e o teste objeivo. Alémdisso, mencionama auto-aveliação, na qual o discente julga seus próprios conhedimentos e suas atitudes. Sua pre sença nos planos poderia ser umdos indicativos de de semperho de habilidades de liderança, mas, na maioria de frequînnia de ocorrênia, os dbcentes aplicaramproves de corheaimertos, não indicandb a presença de formas mais participatives e emancipadoras de avaliação, emconsonânia como djjetivo pretendido de desenvolver umenfermero-líder.

\section{CONSIDERAÇÕES FINAIS}

Ao longo da realização do presente trabalho, pode se constatar que houve umalinhanerto como encontrado por outros conjuntos de pesquisadores preour pados como ensino da liderança. Os adhados deste estudo corroboramo fato, e as autoras ressaltamque o processo de ensino-aprendizagem da Liderança ainda é umgrande desafio para os docentes e discertes da Disciplina de Administração em Enfermagem da FACEN-UNSA

A isso se contrapõe una realidade coma qual esses futuros profissionais estão convivendo: a visão de Barbosa et al. ${ }^{(29)}$ de que, o profissional recebe una formação que não se compatibiliza como que ele encontra no mercado de trabalho atual. 
Opresente estudo tambémdemonstrou que o a míaulo da FACEN-UNSA está vivendiando sucessivas reformilações e mudanças, o que caracteriza ummomerto ided para buscar umensino que supere o que aponta Freire ${ }^{(30)}$ : 0 intelectualismo alienante, 0 atoritaismo do educador e a falsa consciênia do mn do Do contránio, estar-seá, no dzer das a atoras atadas anteriomente ${ }^{(29)}$, apenes naquiandb comu ma nova rour pagemalgo já pronto e estabelecido, pela converiênaia da manutenção do velho ao invés da incônoda insegut rança dos caminhos que leverão ao novo.

Pode-se verificar, tambémque, no bojo dessas mudanças, há esforços dos docentes para fechar essa lacuna, ao dispor, emsua grade arrialar, de uma disciplina eletiva ajo título é OPreparo do Enfermero Líder. Cocreque este esforço é paro prodtivo, porque a disciplina emquestão é oferecida apenas para alguns discentes, sendo de livre escolha e comumnúmero limitado de alunos. Alémdisso, constatourse que a estratégia de ensino predoninante addada pela dbcente responsável por essa disaiplina éa exposição dialogada, complementada pela prática de negociação e comnicação, leitura de apresentação de textos e uso de jogos especáficos.

Algunas dessas estratégias đée podemcontribuir para o desenvolvimento de habilidades de liderança, mas os

\section{Referências}

1. Reltónioparaorecorhedimentodb CursodeGraduacãoemEn fermagem UniversidadedeSantoAmaro. FACGN; 2000.

2. SarmaMC Aevoluçãodadscaplinadeadhinistraçãoadicadaà efermagermaEscoladeE rfermagemdaLSP noperíodode1980 a 1995. RevEscErfLSP 1999; 33(1): 17-30.

3. PozendbC Liderancanaenfermagembrasilera aproximandb-se desua desnistificação. [Dissertação deMestradb] Riberião Preto (SP): EscoladeEremagemdeRibeirãoPreto/田P; 1994.

4. MartinsEG SarmaMC Aproduçãodientíficasodreadhinistração emenfermagermoBrasil no períodb de 1947a 1972. RevBresEff 2005; 58(2): 235-39.

5. Siñ̃esAL, FáveroN OdescilodaliderançaparaoenfermeiroPev Latino-AmErfermagem2003; 11(5): 567-73.

6. Siñ̃es ALde Desenviveropotencial deliderança: Umdesafio parao Enfermeiro[tesededatoradb].RiberãoPreto(\$P): Escola deErfermagemdeRiberiãoPreto/ $\boxplus$ PP; 2001.

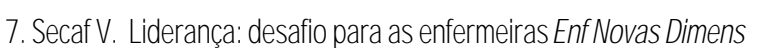
1977; 3(4): 250-55.

8. Sirṽes AL, FáveroN Aprendizagemda Liderança: opinião de enfermerirossobreaformaçãoacadênica. RevLatino-AmEtfemagem 2000; 8(3): 91-96.

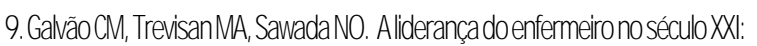
dgumas considaraçoes. PevEscEffư1998; 32(4):302-06. velhos roldes de estratégias comentados anteriomente ainda permanecerme estão distantes desse propósito.

Darte desses adhados levantanos una dérida: será que os dbcentes estão preparados para ensinar como ser um líder? Nimtz e Ciampone ${ }^{(18)}$ salientamque mitas vezes nossos dbcertes se tomammeros repetidores de aulas, perdendb assima dinensão de educadbres e nem sempre se questionamo porquê ensinar e o que ensina.

Coma contribuição que ora se termina de oferecer, abre se una porta para a refleã̃o e ação de nodficação de um a máulo ainda emdesenduimerto, que erfientau diersas rodficasçoes, no qual, os responsávés pelas disaiplines de Adhinistração em Erfermagemsão os professores nis tht ladbs da faculdadee, portanto, emcondção de se comproneteremcamo ensino e a transformaz̧ão do estadb emque este a míalo se encontra Acredtaros que esses resulta dos sinempara un a refleãa, tendb emvista a impatânaja de se formar enfermeros compromissados comaprendza. gemcontína emliderança

Jennings ${ }^{(31)}$ pontua que somos uma sociedade aja principal caraderística é a perda do sentido de autodireção, como está daro na tendênia de fugimmos às responsabilidades da liderança O que se propõe então é que, juntos, discentes e docentes, adquiramo poder da autodireção e assu mama responsabilidade de liderar e formar novos líderes.

10. Chaves日B. Aspectos da liderancanotrabalhodberfermeiro. RevGaúdhaErferm1993; 4(1): 55-58.

11 Hga日R TrevisanMA Osestilos deliderancaidealizados pelos enfermeros RevLatino-AmEtermagem2005; 13(1): 59-64.

12. Recomendações: $020^{\circ}$ Congresso Brasilero deE Efermagem RevBrasErf 1968ago; 21(4): 171.

13. Amdes dela ConferendaLiderzzgodelaEtermeríaen en nuevo milerio. 2000; Mami(USA): ICNFundaciónKellog.

14.Relatóniodb4Encontro Nbaional de Docertes deAdhiristracão emErfermagem 56음esso Brasilero deE fermagem, 2004; Granæab(RS), Brasil. Gramadb(RS): ABEr, 2004.

15-NntzMA Oensinodadsaidiradeadhinistraçãoemefermagermes

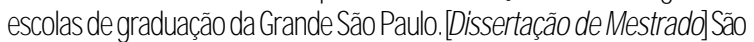

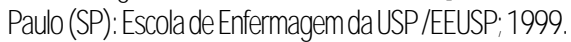

16-RiberioMSartosSL, MeraTGBM Refletindbsobreliderançaem Enfermagem ESC. AnnaNeryRevEnferm. 200610(1): 109-15.

17- SenaRR SilvaK, CoelhoS, BragaPP. Inovaçãonoensinoda enfermagem oUN impulsionandb a diferença Rev. Baiana Erf. 2002; 15(1/2):121-127

18-NmtzMA ClamponeMHT. Osignificadbdacapaditaçãodbcente rapráxis educacional. PevPaulEf 1999; 18(1/3): 27-33.

19- FarialL, CasagrandeLR Aeducaçãopara oséaloXX ea formação do professor reflevivo naenfermagem Revlatino-Am Erfertagem2004;12(5):821-27. 
20 - Gomes JB, Casagrande LRR Aeducação refleviva na pósnodkmidade unarevisãobibliográfica. RevLatino-AmEfremagem 2002; 10(5):696-03.

21-Bordenave JD, PereraAM Estratégias deensino-aprendizagemill. ed. Petó́polis (R)):Vozes; 1989.

22- AbreuMC, MesettoMT. Oprofessor universitánioemaula 9. edSãoPaul(S): 1990

23-MnayoMCS Odesafiodocorhedimento: pesquisaqualitativa emsaúde 7. ed. SãoPaulo(SP): HUTIEC; 2000.

24-BardinL Análisedecontádo.Traducãodel LisAnteroRetoe AugustoPinhero. Edições 70; Lisboa(PT): 1977.

25- CamponeMHT, LeiteMM, Gaicżnski RR Ensinodadisciplina adhinistraçãoemenfermagem embuscadeumnovo paradigna. PevEscErf USP 1996; 30(n esp): 45-58.

\section{Sobre as Autoras}

\section{MíaGistasama}

DoutoraemEremagempda田 $\$$, comPós-datorad emHstória daErermagempelaEANUPBI.

Pesquisadoralndependente Lider do CentrodeEstudos ePesquisa sobreHstóriada E fermagem Gupoindependerte, rãovinaladba qualquer instituiçãodeensimepesquisa.

\section{Loiner Ragiaconstantio}

Aunadb CursodeEspedializaçãoemTerapialintensivadaFaaldade IsraditaAlbetEnstein MentrodoCertrodeEstudosePesquisas emHstóniadaEnfermagemcPPH
26-CasaECCs. OusodePortiólioconrinstrumentodereflexãona formaçãodeenfermeros líderes [dissertaçãodemestrado]. São Palo(SP): UniversidadedeSantoAnaro/UNSA: 2005.

27-SamaMC, arhalCKQ, CamposJP. Estratégiasinovedorasno ensino deadhinistração aplicadaà enfermagem Rev. Paul. Ef. 1999; 18(1/3):16-26.

28-Takdhashi RT Peres HWC Odesafio dau uilização detécricas pedagógicas inovedorasmensinodaadhinistração emerfermagem Pev. Paul. Erf. 2000; 19(2): 20-24.

29-BarbosaMA BrasilW, SasaAL, MonegoEt. Refletindbsobreo desafiodaformaçãodoprofissional dasaúde RevBras Erf2003; 56(5): 57476

30- FreireP. Pedagogiadboprimido. 9. ed. RiodeJanero(P): PazeTera 1981

31-Jernings E Liderançanesargarizaçõesenahistória SãoPalo (SP): Brasiliense, 1970 


\section{ANEXO}

\section{Figral}

TábuadeConvergências dos Disarsos contidos nos "Cbjetivos"

\begin{tabular}{|l|c|}
\hline \multicolumn{1}{|c|}{$\quad$ Calegorias de pensamento } & Freqüêricia de \\
ocorrència
\end{tabular}

